\title{
,
}

\section{A Importância da Comissão de Controle Glicêmico Hospitalar (CCGH) no Hospital Santa Izabel}

\author{
The Importance of the Hospital Glycemic Control Committee \\ (GCHC) at Santa Izabel Hospital
}

\author{
Alina Feitosa ${ }^{1}$ \\ ${ }^{1}$ Serviço de Endocrinologia do Hospital Santa Izabel; Salvador,Bahia, Brasil
}

Correspondence addresses:

Dra. Alina Feitosa

alinafeitosa@yahoo.com.br

Received: April 22, 2019

Revised: May 31, 2019

Accepted: June 18, 2019

Published: June 28, 2019

Data Availability Statement: All relevant data are within the paper and its Supporting Information files.

Funding: This work was the result of author's initiative. There was no support of research or publication funds.

Competing interests: The author has declared that no conflit of interests exists.

Copyright

(C) 2019 by Santa Casa

de Misericórdia da Bahia.

All rights reserved.

ISSN: 2526-5563
Hiperglicemia hospitalar (HH) é uma condição prevalente em hospitais e associada a infecções, maior tempo de internação e mortalidade. Programas estruturados de rastreamento e manejo de glicemias são recomendados por organizações nacionais e internacionais para prover cuidados específicos e reduzir complicações. Em 2017, foi criada a Comissão de Controle Glicêmico Hospitalar (CCGH) no Hospital Santa Izabel com o propósito de prover cuidados específicos para portadores de diabetes e HH. Investimento em preparação, planejamento, equipamentos, tecnologia e pessoal resultou em um programa estruturado de suporte educativo, assistência e vigilância. A CCGH é uma equipe multidisciplinar e montou protocolos, criou workshop de treinamento para colaboradores, impressos educativos para pacientes e reestruturou o serviço nutricional. Acrescentou tecnologia e segurança para o paciente por meio de aviso sonoro da nutrição, prescrição eletrônica de insulinas e interconsulta eletrônica por meio do parecer virtual. $O$ resultado da implantação da CCGH tem sido o maior conhecimento e segurança das equipes na abordagem das HH por meio dos protocolos e ferramentas. Observaram-se redução da média glicêmica, maior uso do esquema basal-bolus de insulina e redução do tempo de internação hospitalar em unidades abertas nos pacientes hiperglicêmicos que tiveram acompanhamento da CCGH quando internados nas unidades fechadas e acompanhados pela equipe de endocrinologia após a alta da unidade fechada.

Palavras-chave: Hiperglicemia; Hospitais; Hospitalização; Unidades de Internação Hospitalar; Insulina.

Hyperglycemia in hospitals $(\mathrm{HH})$ is a prevalent condition in hospitals and associated with infections, more extended hospitalization, and mortality. Structured blood glucose monitoring and management programs are recommended by national and international organizations to provide specific care and reduce complications. In 2017, the Hospital Glycemic Control Committee (HGCC) was created at Santa Izabel Hospital (HSI) to provide specific care for patients with diabetes and $\mathrm{HH}$. Investment in provision, planning, equipment, technology, and personnel resulted in a structured program of educational support, assistance, and surveillance to the HSI. HGCC is a multidisciplinary team that set up protocols, created a training workshop for employees, printed patient education, and restructured the nutritional service. It has added technology and safety for the patient through the sound warning of nutrition, electronic prescription of insulin, and electronic consultation by virtual opinion. The result of the implementation of the HGCC has been the greater knowledge and safety of the teams in the approach of $\mathrm{HH}$ through the protocols and tools. There was a reduction in the glycemic mean, greater use of the basal-bolus insulin regimen, and reduction of hospital admission time in open units in hyperglycemic patients who had HGCC follow-up when admitted to the closed units and followed up by the endocrinology team after discharge from the closed unit. Keywords: Hyperglycemia; Hospitals; Hospitalization; Hospitalization Units; Insulin. 


\section{Epidemiologia da Hiperglicemia Hospitalar}

A hiperglicemia hospitalar é definida por concentrações de glicemia iguais ou maiores que $140 \mathrm{mg} / \mathrm{dL}) .{ }^{1,2}$ Esta condição é encontrada em portadores de diabetes mellitus e em pacientes sem o diagnóstico prévio de diabetes. Nos pacientes sem história prévia de diabetes, a hemoglobina glicada ( $\mathrm{HbA1c}$ ) igual ou superior a $6,5 \%$ confirma o diabetes ${ }^{2}$ e, naqueles sem diabetes prévio ou HbA1C menor do que 6,5\%, são denominados com "hiperglicemia hospitalar ou do estresse". ${ }^{2}$

A hiperglicemia é um transtorno metabólico frequente em hospitais e está relacionada ao maior risco de complicações, custos e mortalidade. ${ }^{3-5}$ É encontrada em $32 \%$ a $38 \%$ dos pacientes em enfermarias, $41 \%$, em portadores de insuficiência cardíaca e doença arterial coronariana e em até $80 \%$ dos pacientes em pós-operatório de cirurgias cardíacas. ${ }^{4,6-8}$ A hiperglicemia está associada ao aumento de mortalidade hospitalar ${ }^{4} \mathrm{e}$ a inúmeras morbidades como insuficiência renal aguda, infecções, ${ }^{9}$ síndromes coronarianas agudas, desidratação, distúrbios eletrolíticos e reinternação. ${ }^{10}$ Adicionalmente, o tratamento das hiperglicemias por meio de insulinas aumenta a chance de hipoglicemias, o que tem sido associado ao aumento do tempo de internação hospitalar, complicações neurológicas, eventos isquêmicos e mortalidade. ${ }^{11}$

A despeito da elevada prevalência e dos efeitos deletérios comprovados em pacientes hospitalizados, frequentementea hiperglicemia é negligenciada ou tratada de forma inadequada. ${ }^{12}$ A hipoglicemia é temida pelos profissionais de saúde, não é adequadamente abordada e prevenida e, consequentemente, limita a insulinização apropriada. $\mathrm{O}$ cenário usualmente encontrado em hospitais é de subdiagnóstico das hiperglicemias, falta de monitoração, desconhecimento sobre o tratamento e os protocolos institucionais e falha no seguimento das recomendações e diretrizes específicas. ${ }^{13,14}$
O resultado da falta de abordagem apropriada das disglicemias (hiper e hipoglicemias) hospitalares é o descontrole glicêmico. Estudo brasileiro revelou que a maioria dos pacientes portadores de diabetes encontram-se severamente descompensados durante uma internação hospitalar. Dentre 2.399 pacientes de 24 hospitais em 13 cidades brasileiras, $89,4 \%$ e $88,2 \%$ tinham glicemias acima de $180 \mathrm{mg} / \mathrm{dL}$ e 30,9 e $27,3 \%$ tinham glicemias inferiores a $70 \mathrm{mg} / \mathrm{dL}$, em unidades de terapia intensiva e enfermarias, respectivamente. ${ }^{15}$

\section{Metas Glicêmicas em um Hospital}

A despeito da associação entre disglicemias e maus desfechos, há controvérsias sobre o impacto do controle glicêmico para pacientes e em indicadores hospitalares assim como não é consenso qual a meta glicêmica ideal para o ambiente hospitalar. ${ }^{16}$

A maioria das diretrizes recomenda o alvo glicêmico entre $140 \mathrm{mg} / \mathrm{dL}$ e $180 \mathrm{mg} / \mathrm{dL}$ em ambiente crítico e inferiores a $140 \mathrm{mg} / \mathrm{dL}$ e $180 \mathrm{mg} / \mathrm{dL}$ para glicemias pré-prandiais e aleatórias, respectivamente, em unidades abertas. ${ }^{1,17}$ A despeito de não haver diferença na mortalidade entre glicemias inferiores a $110 \mathrm{mg} / \mathrm{dL}$, entre $110 \mathrm{mg} / \mathrm{dL}$ a $140 \mathrm{mg} / \mathrm{dL}$, entre $140 \mathrm{mg} / \mathrm{dL}$ a $180 \mathrm{mg} / \mathrm{dL}$ e superior a $180 \mathrm{mg} / \mathrm{dL}$, valores inferiores a $110 \mathrm{mg} / \mathrm{dL}$ e entre $110 \mathrm{mg} / \mathrm{dL}$ a $140 \mathrm{mg} / \mathrm{dL}$ estão associados a maior risco de hipoglicemias. ${ }^{18}$ Embora haja carência de dados definitivos sobre os alvos glicêmicos ótimos ${ }^{17}$ devido ao pequeno número de ensaios clínicos, considera-se o alvo de $140 \mathrm{mg} / \mathrm{dL}$ a $180 \mathrm{mg} / \mathrm{dL}$ como suficiente para melhorar desfechos clínicos em cenários críticos. ${ }^{1,17}$ Alvos mais rigorosos, entre $110 \mathrm{mg} / \mathrm{dL}$ a $140 \mathrm{mg} / \mathrm{dL}$, podem ser apropriados para pacientes selecionados desde que sejam alcançados sem significativo aumento do risco de hipoglicemias. ${ }^{17}$

Quanto ao impacto do controle glicêmico nos desfechos, há controvérsias. Em pacientes internados em unidades abertas, faltam estudos 
definitivos, entretanto trabalhos revelam benefícios do controle glicêmico, como a redução de complicações, ${ }^{19}$ reinternações ${ }^{20}$ e do tempo de internação em unidades abertas e fechadas. ${ }^{21}$

\section{Programas de Controle Glicêmico Hospitalar}

Segundo a Sociedade de Medicina Hospitalar, ${ }^{16}$ evidências incompletas ou imperfeitas não devem ser uma desculpa para falta de ações ou a adoção de padronizações inaceitáveis que têm se mostrado inefetivas e, eventualmente, potencialmente perigosas para o paciente. A Associação Americana de Endocrinologistas Clínicos reforça que a falta de evidências definitivas não endossa uma abordagem casual do controle glicêmico hospitalar. ${ }^{22}$

Organizações de profissionais de saúde envolvendo cuidados hospitalares em portadores de disglicemias, incluindo a Sociedade Brasileira de Diabetes (SBD), Associação Americana de Diabetes (ADA), Associação Americana do Coração (AHA), Associação Americana de Educadores em Diabetes (AADE), Associação Americana de Endocrinologistas Clínicos (AACE), Sociedade de Endocrinologia(Endocrine Society), Sociedade Europeia de Endocrinologia (ESE) e a Sociedade de Medicina Hospitalar $(\mathrm{SMH})$ proveem recomendações de como alcançar alvos glicêmicos seguros por meio de protocolos, procedimentos e melhorias sistêmicas para facilitar a implantação de programas específicos.

Entretanto, a despeito dos significativos esforços para melhorar o controle glicêmico hospitalar na última década, danos resultantes das hipoglicemias e hiperglicemias continuam persistentes e permanecem muito comuns. ${ }^{15,23}$ Uma das mais importantes limitações é a insulinoterapia. Para o tratamento de hiperglicemias, recomenda-se a prescrição de esquema basal-bolus de insulina, que resulta em menor média glicêmica, embora aumente a taxa de hipoglicemias leves. ${ }^{2,4}$ No entanto, mesmo sendo a principal medicação para tratar as hiperglicemias hospitalares, a insulinoterapia é, frequentemente, prescrita de forma inapropriada ou errada. O programa de erro de medicações americano relata que a insulina é responsável por mais de 50\% dos erros reportados, destacando-se a omissão e doses inapropriadas. ${ }^{25}$

Com o propósito de prevenir e reduzir os danos das disglicemias hospitalares, organizações de saúde internacionais ${ }^{2,16,17}$ e a Sociedade Brasileira de Diabetes recomendam implantação de programas de controle de glicemias em pacientes hospitalizados. ${ }^{26}$ Programas hospitalares de manejo de doenças são importantes componentes de sistemas de saúde complexos, pois servem para coordenar os cuidados crônicos de saúde, promover detecção precoce de problemas e para prevenir e reduzir os custos em saúde. Ainda que um programa estruturado seja o formato ideal de abordagem das disglicemias, a presença de programas parciais, com apenas um time de diabetes, já resulta em redução do tempo de internação. ${ }^{27-29}$

Renomados pesquisadores propõem que a metodologia de melhoria da qualidade de programas de controle de infecção hospitalar sirva como modelo de excelência para a melhoria da qualidade em programas hospitalares de controle glicêmico. ${ }^{30} \mathrm{Na}$ busca pela otimização de processos nos cuidados com as glicemias, há certificações internacionais específicas para diabetes intra-hospitalar como a The Joint Commision Disease Specific Care Certification, focadas no cuidado clínico e relação médicopaciente, baseadas no modelo centrado no paciente $^{31}$ e que propõem uma forma de organizar os cuidados em etapas. Certificações e acreditações são instrumentos essenciais na profissionalização da gestão, redução de custos e busca pela excelência.

\section{Hospital Santa Izabel e um Programa de Controle Glicêmico Hospitalar}

O Hospital Santa Izabel reconheceu a importância e a necessidade de um programa específico de cuidados com as disglicemias hospitalares e com o diabetes. Em agosto de 2017, apoiou a iniciativa da criação da Comissão de 
Controle Glicêmico Hospitalar (CCGH) provendo expressivo investimento em preparação, planejamento, tempo, equipamentos, tecnologia e pessoal, resultando em um programa estruturado de suporte educativo, assistência e vigilância. A primeira unidade em que foi implantada a $\mathrm{CCGH}$ foi a UTI cardiovascular em abril de 2018. Seguiram-se as UTIs cirúrgica, neuroclínica, unidade coronariana e UTI clínica adulta.

A criação da CCGH seguiu as recomendações do guia de implantação de controle glicêmico hospitalar da Sociedade de Medicina Hospitalar ${ }^{16}$ e inspirou-se, com adaptações e inovações, no hospital paulista Albert Einstein, que tem certificação da Joint Commission International para cuidados com portadores de diabetes.

São considerados elementos essenciais para a implantação de uma $\mathrm{CCGH}:{ }^{16}$

- Obter suporte institucional;

- Criar uma equipe multidisciplinar;

- Desenvolver objetivos específicos ou metas que são tempo-definidas, mensuráveis e alcançáveis;

- Acessar o status do manejo glicêmico da instituição;

- Revisar e identificar a melhor forma de manejo do diabetes e hiperglicemias no hospital;

- Escolher a métrica e coletar dados;

- Desenvolver processos e estruturação para prevenir hipoglicemias;

- Desenvolver e implementar protocolos e algoritmos "instituição-específicos" para tratamento das hiperglicemias e monitorar o impacto da intervenção; e

- Mudar e manter a nova cultura da melhoria.

\section{A Comissão de Controle Glicêmico do Hospital Santa Izabel - CCGH do HSI}

Seguindo as recomendações do guia para implantação de um programa de controle glicêmico hospitalar, foi criada uma comissão multidisciplinar composta por enfermeiras, farmacêuticas, bioquímicas, nutricionistas, engenheiro clínico, analista de sistemas e analistade processos, profissional da comunicação e marketing, gerente de suprimentos e médico endocrinologista (Tabela 1).

As ações que se seguiram à montagem da equipe foram:

1. Reuniões para integração e planejamento de ações;

2. Estabelecer a vigilância epidemiológica do controle glicêmico;

3. Estabelecer metas e etapas a serem cumpridas;

4. Explorar as barreiras e montar estratégias educativas para pacientes, acompanhantes e profissionais de saúde;

5. Construção de protocolos, procedimentos e processos para o controle glicêmico;

6. Ordens médicas eletrônicas;

7. Ajustar a terapia nutricional: criação de cardápios especiais, book ilustrativo e folder educativo;

8. Criação de ferramentas para vigilância: relatórios periódicos do cenário glicêmico e interconsultoria virtual;

9. Substituição dos glicosímetros por equipamentos de acordo com a resolução da ANVISA, RDC 302.

Reuniões para Integração e Planejamento de $\underline{\text { Ações }}$

Reuniões periódicas com a equipe multidisciplinar são realizadas para o planejamento de ações, estudos das barreiras e criação de soluções.

Estabelecer a Vigilância Epidemiológica do Controle Glicêmico

Estudo do cenário glicêmico do HSI antes da implantação e permanentemente.

\section{Avaliação do Cenário Glicêmico}

Foram estudadas as internações de adultosentre janeiro de 2016 a junho de 2017 ( $n=37.624)$.

As diretrizes preconizam a realização do rastreamento da hiperglicemia por meio da glicemia à admissão. Avaliamos o percentual de indivíduos que realizaram pelo menos uma glicemia capilar dentre as primeiras 24 horas 
Tabela 1. Equipe da CCGH.

\begin{tabular}{ll}
\hline Colaborador da Equipe & Cargo \\
\hline Allyson Bortoli & Engenheiro - Supervisor da Engenharia Clínica \\
Alina Feitosa & Médica Endocrinologista - Supervisora da CCGH \\
Ângela Alencar & Enfermeira - Coordenadora da Conde 1 e UTP \\
Aridea Mamédio & Enfermeira - Coordenadora das UTIs cardiovascular e da UCO \\
Carla Oliveira & Enfermeira - Coordenadora da UTI Pediátrica \\
Carina Aleixo & Enfermeira - Desenvolvimento de Pessoas \\
Cláudio Reis & Coordenador - Processos e Sistemas \\
Cristiane Hoffmeister & Farmacêutica - Coordenadora Geral \\
Darci Malaquias & Nutricionista - Coordenadora da Nutrição Clínica e Terapia Nutricional \\
Jacqueline Noronha & Enfermeira - Gerente de Assistência \\
Jaqueline Dourado & Enfermeira - Desenvolvimento de Pessoas \\
Jeanny Barreto & Enfermeira - Coordenadora da UTI Cirúrgica \\
Jussara Silveira & Bioquímica - Supervisora Técnica do Laboratório \\
Lígia Wange & Enfermeira - Gerente de Assistência \\
Lorena Sales & Supervisora - Marketing e Comunicação \\
Lorena Cordeiro & Nutricionista \\
Manuella Trimailovas & Bioquímica - Coordenadora do Laboratório \\
Natalia Cairo Leal & Farmacêutica Clínica \\
Maria Neila Almeida & Nutricionista - Coordenadora de Produção \\
Valterney Andrade & Analista de Processos \\
Rafael Castro & Gerente de Suprimentos \\
Sayuri Yamashita & Farmacêutica Clínica \\
Thais Mirelle & Enfermeira - Desenvolvimento de Pessoas \\
Wellington Fava & Analista de Processos \\
Yanderson Cavalcante & Analista de Sistemas \\
\hline
\end{tabular}

após a admissão hospitalar. Dentre o total de 13.574 glicemias capilares realizadas $(36,1 \%$ dos pacientes internados), 11.578 foram feitas nas primeiras 24 horas. Considerando-se a população internada, apenas $30,8 \%$ realizaram o rastreamento nas primeiras $24 \mathrm{~h}$.

Buscando-se os códigos internacionais de doenças compatíveis com diabetes preenchidos em formulários de admissão, alta e em antecedentes médicos, identificou-se a prevalência de diabetes em apenas $2,53 \%$ dos internados, incongruente com a realidade e compatível com subnotificação do diagnóstico.

Dentre os pacientes que realizaram pelo menos uma glicemia, $41,1 \%$ apresentavam valores maiores que $140 \mathrm{mg} / \mathrm{dL}, 2,1 \%$, menores que $70 \mathrm{mg} / \mathrm{dL}$ e $15,2 \%$, acima de $200 \mathrm{mg} / \mathrm{dL}$.
Ao avaliar a relação entre a primeira glicemia capilar realizada e o tempo de internação, observou-se uma distribuição de tempo de internação do tipo curva J, com maior duração de internação quando as glicemias eram inferiores a $70 \mathrm{mg} / \mathrm{dL}$ e superiores a $140 \mathrm{mg} / \mathrm{dL}$ (Figura 1).

A primeira avaliação feita pela CCGH revelou cenário de subnotificação do diagnóstico de diabetes, rastreamentodeficiente dahiperglicemia hospitalar e maior duração de internação em pacientes com hipo e hiperglicemias.

Estabelecer Metas e Etapas a Serem Cumpridas A CCGH tem como metas:

- Melhoria dos processos associados ao reconhecimento e manejo de disglicemias hospitalares; 
- A educação continuada e motivação da equipe de assistência;

- Melhoria da experiência do paciente;

- Redução de infecções, custo, tempo de internação hospitalar e taxa de reinternações;

- Valorização do hospital perante as operadoras de saúde como um modelo excelente de assistência doença-específica;

- Receber certificação doença-específica como o selo "Hospital Amigo do Diabetes", um selo em planejamento da Sociedade Brasileira de Diabetes para hospitais que cumprirem requisitos de assistência aos diabéticos.

As diretrizes da Sociedade de Medicina Hospitalar salientam que resultados com desfechos relacionados a um programa estruturado devem ser esperados após 12 a 18 meses da implantação. ${ }^{16}$

Explorar as Barreiras e Montar Estratégias Educativas para Pacientes, Acompanhantes e Profissionais de Saúde

\section{Explorar as Barreiras - Inquérito da CCGH}

A CCGH realizou um inquérito* para avaliação de conhecimento, percepção e barreiras relacionadas ao manejo glicêmico hospitalar.

Uma amostra de $50 \%$ dos colaboradores médicos, médicos-residentes, enfermeiros, técnicos de enfermagem e nutricionistas que atuassem em unidades abertas adultas, UTI clínica e cirúrgica adultas foi, aleatoriamente, selecionada. Foi criado um instrumento com questões de múltipla escolha baseado em questionários utilizados em estudos semelhantes e aplicado por entrevistadores treinados.

Foram entrevistados 89 colaboradores e, dentre os resultados encontrados, o que mais chamou a atenção foi o desconhecimento da definição de hiperglicemia hospitalar. Apenas $26,1 \%$ dos entrevistados sabiam o valor que define a hiperglicemia hospitalar (Figura 2).

Desenvolvimento de Materiais Educativos para Pacientes e Acompanhantes

Foram criadas a cartilha e o folder educativo sobre hiperglicemia hospitalar, com informações sobre o diagnóstico, impacto e tratamento das hiperglicemias por meio de ilustrações e linguagem para o público leigo. Esses materiais são fornecidos aos pacientes com diabetes mellitus prévio e hiperglicemia hospitalar.

Também foram criados os impressos para educação e proteção do portador de diabetes: cartão do diabético, folha de registro de glicemias capilares, receituário padronizado de insulinas e o formulário de orientações sobre uso de insulinas na alta que ficam disponíveis na pasta da $\mathrm{CCGH}$,

Figura 1. Tempo de internação e faixas de glicemias $(\mathrm{n}=13.547)$.

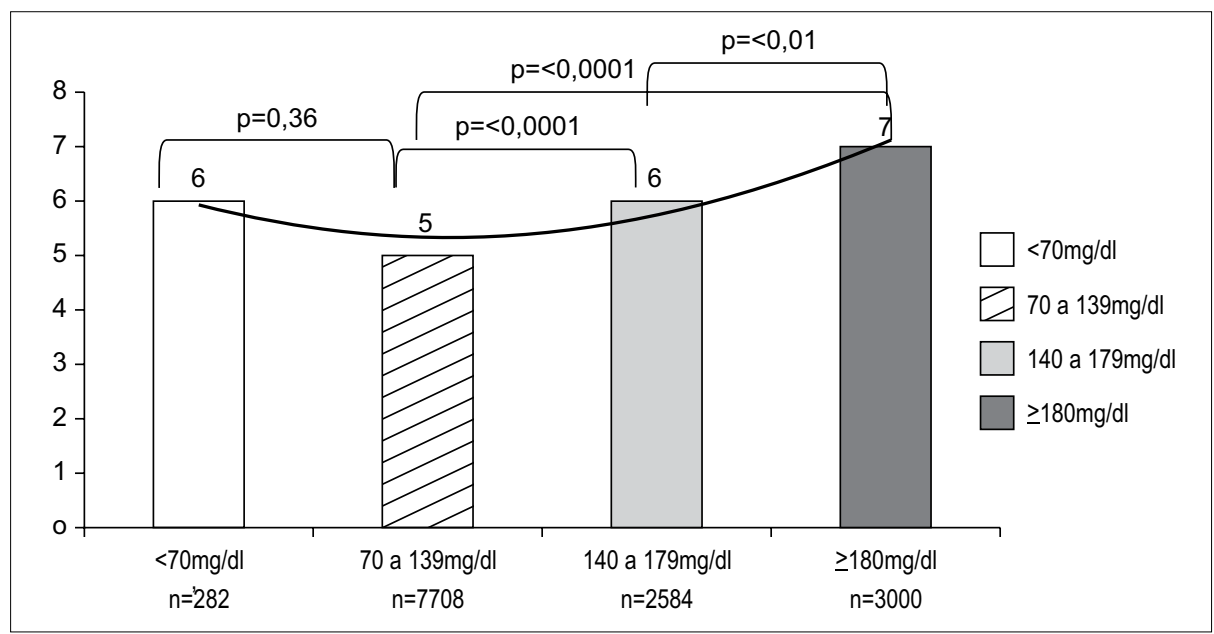

*Entrevistadora que conduziu a avaliação: Dra. Ana Carolina Matos, médica endocrinologista, que foi residente de clínica médica do Hospital Santa Izabel em 2013. 
Figura 2. Valores que definem hiperglicemia hospitalar segundo colaboradores de saúde.

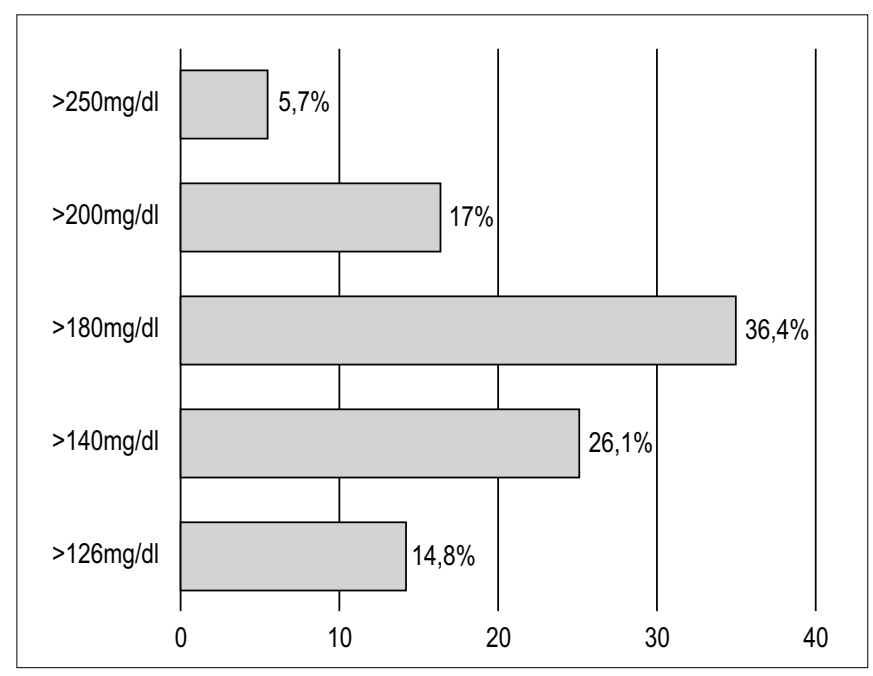

na área de trabalho de todos os computadores do HSI (Figura 3).

Construção de Protocolos, Procedimentos e Processos para o Controle Glicêmico

\section{Protocolos da CCGH}

Foram criados protocolos de conduta na admissão hospitalar, insulinização subcutânea e de transição, insulinização venosa e tratamento de hipoglicemias. Os protocolos criados basearam-se nas recomendações das diretrizes e algoritmos nacionais, internacionais e reuniões com coordenadores médicos e enfermeiras para adaptar os algoritmos às rotinas das diferentes unidades. Os protocolos estão disponíveis em pasta denominada CCGH, disponível na área de trabalho de todos os computadores do HSI.

Para a insulinização venosa, a $\mathrm{CCGH}$ recomenda o uso do aplicativo insulinapp UTI (Figura 4). ${ }^{32} \mathrm{O}$ link para o aplicativo fica disponível na área de trabalho e o aplicativo auxilia a equipe de saúde na insulinização por via endovenosa calculando a velocidade de infusão da solução de insulina hora a hora. Os ajustes da sensibilidade à insulina são feitos por meio de algoritmos que interpretam os valores de glicemia e as variações hora a hora e resultam em categorias de sensibilidade. Ao inserir os dados de glicemia e categoria, o aplicativo fornece a nova velocidade de infusão. O manejo do aplicativo e a modificação da velocidade de infusão são executados pela enfermagem e os dados gerados são inseridos em formulário no PEP.

Este aplicativo foi desenvolvido por médicos endocrinologistas e validado por enfermeiros e médicos intensivistas, promovendo o alcance de alvo glicêmico (140mg/dL a $180 \mathrm{mg} / \mathrm{dL}$ ) em uma mediana de 7 horas de uso, com baixo risco de hipoglicemias. ${ }^{33}$

\section{Workshop de Insulinoterapia e Controle Glicêmico Hospitalar}

Foi criado um curso teórico-prático com simulações realísticas para colaboradores a fim de prover treinamento e educação continuada para o controle glicêmico hospitalar. O público alvo são médicos, enfermeiros, técnicos de enfermagem, nutricionistas e farmacêuticos. O curso tem duração de 3 horas e é ministrado pela supervisora e três enfermeiras do setor de desenvolvimento de pessoas. É composto de avaliação com questões de múltipla escolha aplicado antes e após o curso, aula teórica, questões práticas e simulações realísticas para treinamento do reconhecimento da importância, problemas, rastreamento, diagnóstico e tratamento das disglicemias hospitalares. As atividades de treinamento são realizadas em 
Figura 3. Folders para educação e proteção do portador de diabetes.

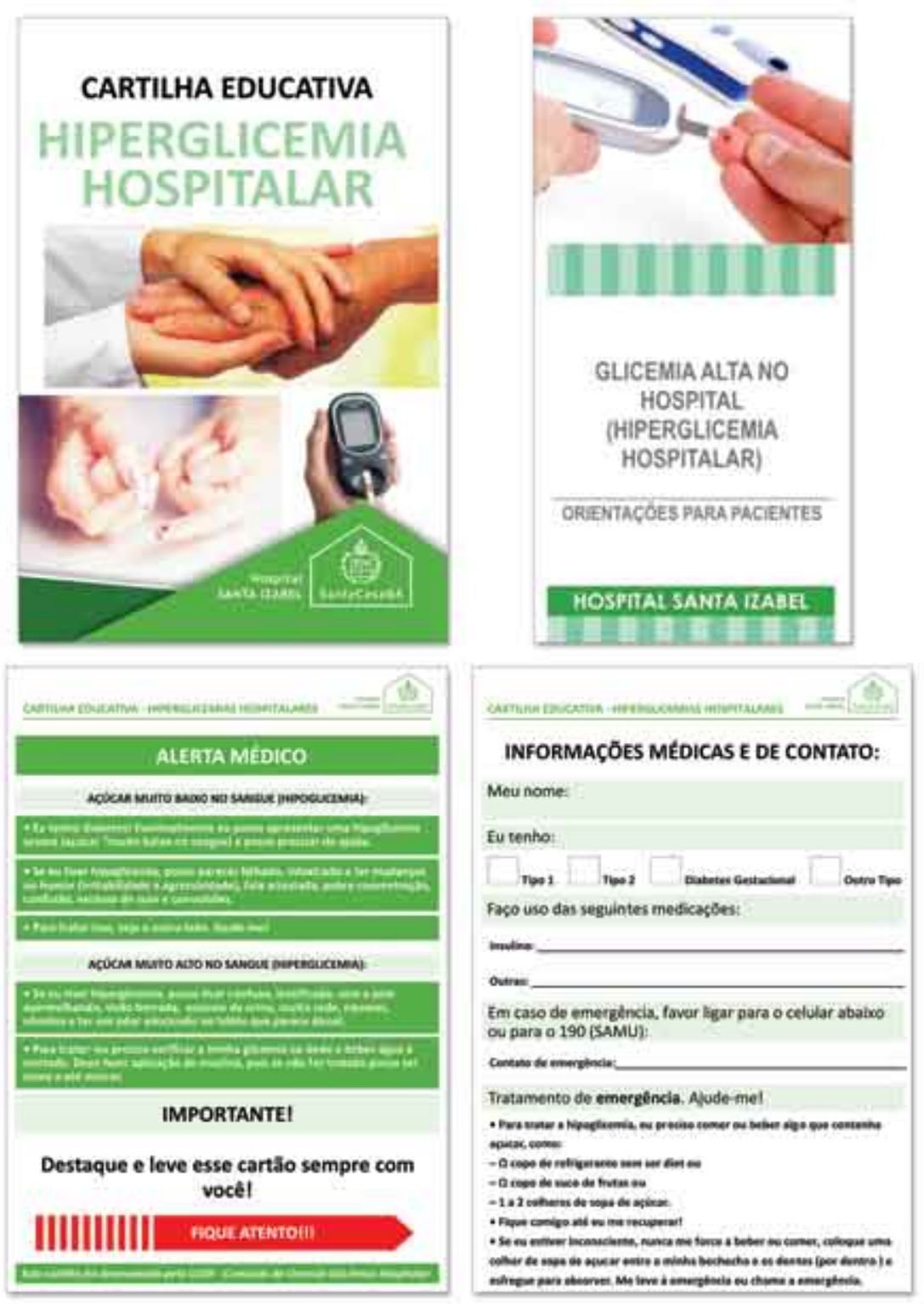

Figura 4. InsulinAPP-UTI - Tela Inicial.

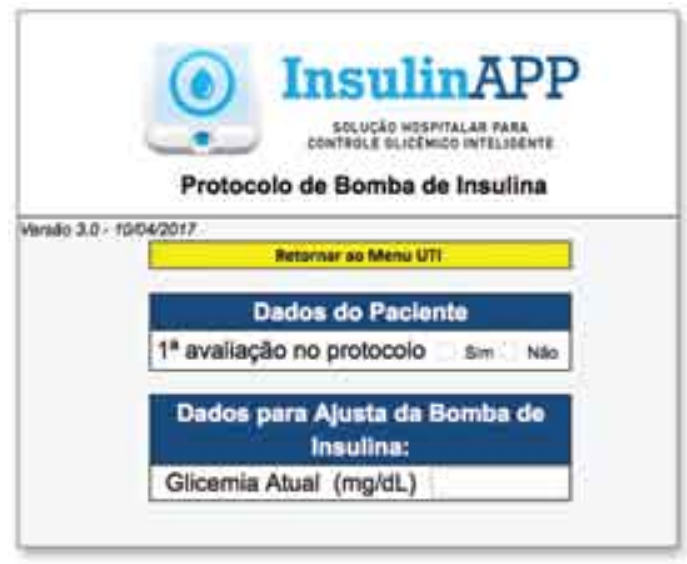


Tabela 2. Quantitativo de participantes do Workshop da CCGH por categoria de colaborador de saúde.

\begin{tabular}{lcccccc}
\hline & \multicolumn{6}{c}{ Quantitativo de Participantes do Workshop da CCGH por Categoria } \\
\cline { 2 - 7 } Mês/Ano & Enfermagem & Médicos & Nutricionistas & Farmacêutica & Membros CCGH & Total \\
\hline Set/17 & 0 & 9 & 0 & 0 & 11 & 20 \\
Mar/18 & 35 & 1 & 3 & 0 & 0 & 39 \\
Abr/18 & 6 & 0 & 0 & 0 & 0 & 6 \\
Jul/18 & 53 & 2 & 0 & 1 & 0 & 55 \\
Nov/18 & 30 & 2 & 0 & 0 & 0 & 32 \\
Jan/19 & 54 & 2 & 0 & 1 & 11 & 208 \\
Total & 178 & 16 & 3 & & & 56 \\
\hline
\end{tabular}

Figura 5. Percentual de acertos no teste pré- e pós-Workshop da CCGH.

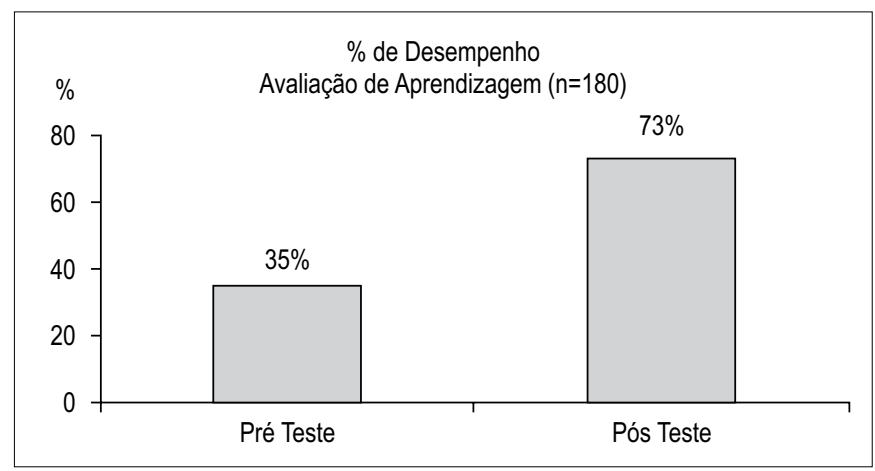

grupos com 5 a 6 pessoas, sempre compostos por equipe multidisciplinar.

$\mathrm{O}$ treinamento já foi realizado em 5 unidades fechadas e 208 colaboradores foram treinados (Tabela 2).

Verificamos melhoria dos conhecimentos sobre hiperglicemias hospitalares na avaliação após o workshop (Figura 5).

O planejamento futuro é repetir, periodicamente, a avaliação teórica naqueles que já fizeram o workshop para verificar a necessidade de novo treinamento e, permanentemente, treinar os médicos egressos à instituição, incluindo os médicos-residentes, que se renovam anualmente.

\section{Aviso Sonoro da Nutrição}

A terapia nutricional é importante para otimizar o controle glicêmico e prover adequada quantidade de calorias e nutrição para a demanda metabólica. Em pacientes com hiperglicemia, o tratamento mais utilizado no hospital é a insulinoterapia. A periodicidade e o momento em que a insulina é administrada depende da forma como o paciente é alimentado: se alimentado por via oral, deve ser utilizada 15 a 30 minutos antes das refeições; se por via enteral, parenteral ou em jejum, deve ser administrada a cada 4 ou 6 horas.

Enquanto a maioria das medicações prescritas são aprazadas para horários fixos, a administração da insulina deve estar relacionada à refeição. Idealmente, a insulina pré-refeição não deve ser aprazada. A refeição precisa chegar à unidade, ser distribuída e a insulina aplicada antes do início da refeição. Essa é uma das tarefas mais difíceis de cumprir-se no hospital: garantir a adequada sincronia entre a administração da insulina antes da alimentação em pacientes alimentados por via oral.

As insulinas ultra rápida (lispro, aspart egluilisina) e rápida (regular) devem ser aplicadas 
15 e 30 minutos antes do início da refeição, respectivamente, e, usualmente, a falta dessa sincronia gera variabilidade glicêmica, aumentando o risco de hipo e hiperglicemias. O problema é que não há um horário fixo para a saída do carrinho de alimentos da nutrição produção e tampouco a precisão na hora de chegada às unidades, dificultando a coordenação entre medicação e alimentação.

Para resolver este problema, a CCGH inovou, por meio da tecnologia, criando o aviso sonoro da nutrição para alertar à unidade que o carrinho saiu da produção e chegaráà unidade em $5 \mathrm{a} 10$ minutos. $\mathrm{O}$ aviso permite que a enfermagem mensure a glicemia e administre as insulinas prescritas antes da chegada prevista das refeições.

$\mathrm{O}$ aviso sonoro é constituído de cinco notas musicais curtas seguidas de um anúncio em voz feminina: "nu-tri-ção", que se repete por quatro vezes separadas por um minuto. $\mathrm{O}$ som é propagado em toda a unidade (Figura 6). $\mathrm{O}$ paciente também fica ciente do som e participa do processo aprendendo e reforçando o uso da insulina antes das refeições.

$\mathrm{O}$ processo de acionamento do aviso sonoro é feito pela funcionária que opera o carrinho de alimentos: 1. Ao sair da nutrição produção, faz a discagem de um código e o número de ramal da unidade alvo; 2. A seguir, desloca-se até a unidade alvo.

$\mathrm{O}$ aviso foi recebido com surpresa e alegria pelos colaboradores das unidades fechadas que conseguem, atualmente, manter a sincronia das insulinas com as refeições, obedecendo à farmacodinâmica adequada das insulinas, minimizando erros, variabilidades nas glicemias e eventos adversos.

\section{Ordens Médicas Eletrônicas}

\section{Prescrição Eletrônica de Insulinas}

Diretrizes recomendam o uso de ordens médicas computadorizadas com a finalidade de prevenir os erros relacionados à prescrição e para aumentar a eficiência da administração da medicação. ${ }^{34}$ Revisão de ensaios clínicos randomizados da Fundação Cochrane, que avaliaram ordens eletrônicas para controle glicêmico hospitalar, demonstrou significativa melhora do percentual do tempo em alvo, menor média glicêmica sem aumento de hipoglicemias. ${ }^{35}$

Em conjunto com a Tecnologia de Informática (TI) do Hospital Santa Izabel e em congruência com as recomendações de incorporação de todos os componentes da insulinoterapia estruturada

Figura 6. Esquematização do funcionamento do aviso sonoro da nutrição - CCGH.

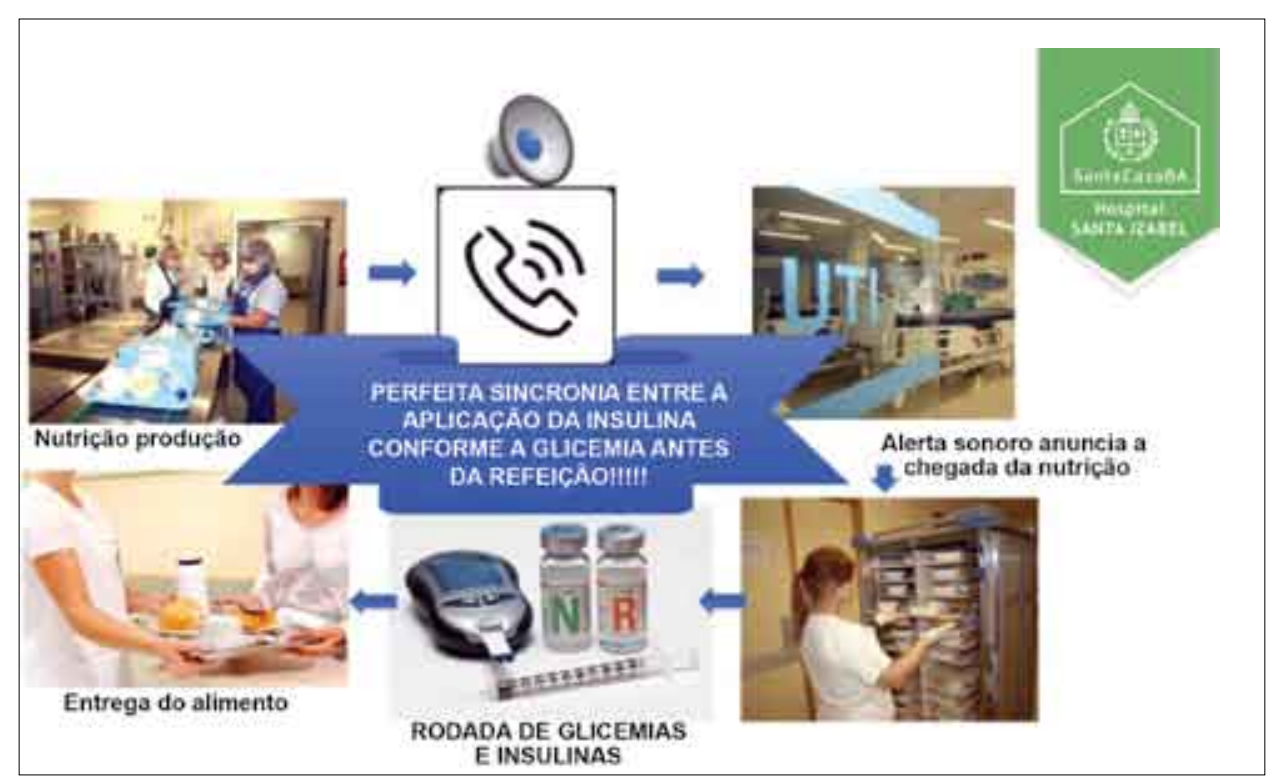


na prescrição eletrônica, a CCGH criou uma ferramenta para prescrição eletrônica de insulinas dentro do PEP. A estrutura da ferramenta baseouse no modelo de prescrição eletrônica de insulinas da Sociedade de Medicina Hospitalar. ${ }^{36}$

Ao utilizar a prescrição eletrônica de insulinas, o médico prescreve de acordo com as recomendações e algoritmos das diretrizes de manejo glicêmico hospitalar, e as doses de insulinas são determinadas por cálculos da dose por quilo de peso e características clínicas do paciente. A forma de administração de insulina, a monitoração das glicemias capilares e o tratamento de hipoglicemias (glicose venosa, açúcar por via oral e glucagon intramuscular) são automaticamente gerados na prescrição após o preenchimento de curto formulário dentro do PEP (Figura 7).

\section{Tutorial para Treinamento em Prescrição Eletrônica de Insulinas}

Foi criado um documento para auxiliar os prescritores no manejo da prescrição eletrônica de insulinas. O tutorial fica disponível na pasta CCGH, na área de trabalho, e foi repassado aos plantonistas e coordenadores das unidades fechadas.
Treinamento Prático para Prescrição Eletrônica de Insulinas

As farmacêuticas da CCGHrealizaram o primeiro treinamento para prescrição eletrônica de insulinas em maio de 2019, quando foram treinados 28 médicos plantonistas das unidades UTI clínica, UTI cirúrgica,UTI cardiovascular,Unidade deneurologia e Unidade coronariana. O treinamento aconteceu dentro das próprias unidades, durou cerca de 15 minutos, com o tutorial de prescrição eletrônica de insulinas e o computador para simulação. O treinamento dos plantonistas das unidades fechadas durou uma semana e foi realizado nos turnos da manhã e tarde. O planejamento futuro é realizar o treinamento com os médicos das unidades abertas e, periodicamente, treinar os médicos egressos ao hospital.

\section{Formulários Eletrônicos de Suporte à Terapia} com Insulinas: Receituário de Insulinas, Folha de Registros de Glicemias, Orientações aos Usuários de Insulinas

Modelo de receituário de insulina, folha para registro de glicemias para que o paciente possa registrar as suas glicemias e acompanhar o tratamento e orientações escritas sobre insulinas, forma de administração e acondicionamento

Figura 7. Formulário para prescrição eletrônica de insulinas no PEP.

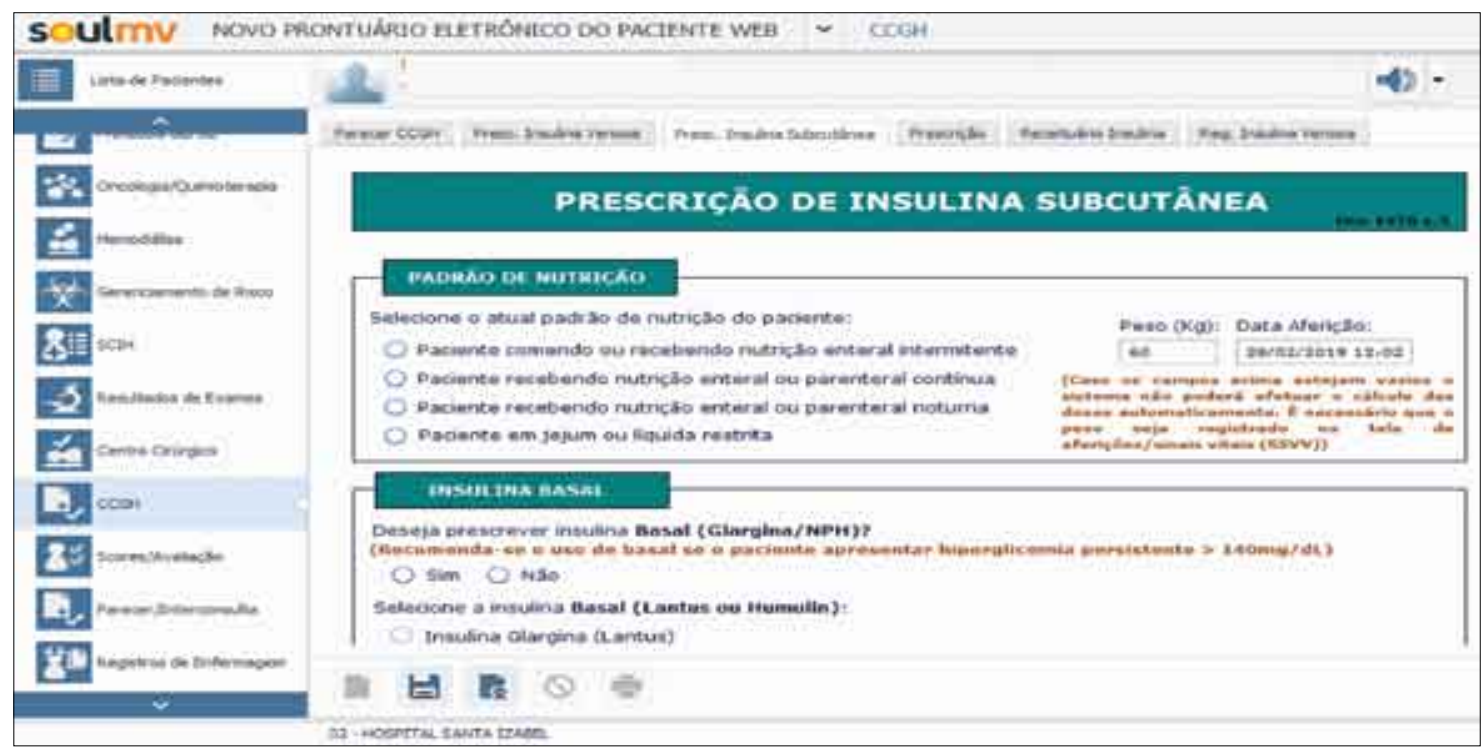


ficam disponíveis na pasta da CCGH, na área de trabalho, para auxilio na educação do paciente.

Ajustar a Terapia Nutricional: Criação de Cardápios Especiais, Book Ilustrativo e Folder Educativo

Para a abordagem de portadores de hiperglicemias hospitalares é preciso ter um cardápio especial que contemple composição com menor proporção de carboidratos, carboidratos complexos provindos de alimentos integrais e enriquecidos com fibras e, preferencialmente, quantidade fixa de carboidratos por refeição, para a adequada proporção entre insulina e carboidratos.

Aequipe de nutrição produção, nutrição clínica e endocrinologia da CCGH analisaram os cardápios existentes e os alimentos disponíveis na produção e criaram 5 cardápios com 6 refeições com os totais calóricos diários de 1.500, 1.800, 2.000, $2.200 \mathrm{e}$ $2.500 \mathrm{kcal} /$ dia para suprir as demandas metabólicas dos diversos pacientes (Figura 8).
Cada refeição desses cardápios tem a quantidade fixa de carboidratos permitindo o ajuste de insulinas em doses fixas com menor variabilidade pós-prandial, conforme a recomendação das diretrizes. A substituição de parte dos carboidratos por proteínas também foi contemplado, o que aumenta os custos do cardápio especial, mas com o entendimento que resultaria em melhorias no controle glicêmico e educação do paciente.

É uma barreira para pacientes a aceitação de mudanças de seus padrões alimentares habituais $\mathrm{e}$, frequentemente, pacientes $\mathrm{e}$ familiares questionam e reclamam sobre a quantidade, o tipo de alimento e não entendem a alimentação do hospital como parte da terapia. Há pacientes que desrespeitam as normas hospitalares e consomem alimentos trazidos por familiares, dificultando o controle de suas glicemias. Para minimizar essas barreiras, aumentar a aderência, o entendimento e a satisfação do paciente, a $\mathrm{CCGH}$, com a nutrição montou um livro com

Figura 8. Cardápios especiais com quantidade fixa de carboidratos.

\begin{tabular}{|c|c|c|c|c|c|c|}
\hline \multirow{7}{*}{ DESJEJUM } & Café preto - $100 \mathrm{~mL}$ & Café preto $-100 \mathrm{~mL}$ & Café preto - 150mL & Café preto - 150mL & Café preto - 150mL & Café - leite \\
\hline & Leite desn - $100 \mathrm{~mL}$ & Leite desn $-100 \mathrm{~mL}$ & Leite desn - $150 \mathrm{~mL}$ & Leite desn - $150 \mathrm{~mL}$ & Leite desn - $150 \mathrm{~mL}$ & Leite - café \\
\hline & $\begin{array}{l}\text { Queijo branco - } \\
\text { 30g OU ovo - } 2 \\
\text { unidades }\end{array}$ & $\begin{array}{l}\text { Queijo branco - } \\
60 \mathrm{~g} \text { OU ovo - } 2 \\
\text { unidades }\end{array}$ & $\begin{array}{l}\text { Queijo branco - } \\
60 \mathrm{~g} \text { OU ovo - } 2 \\
\text { unidades }\end{array}$ & $\begin{array}{l}\text { Queijo branco - } \\
\text { 90g OU ovo - } 2 \\
\text { unidades }\end{array}$ & $\begin{array}{l}\text { Queijo branco - } \\
\text { 90g OU ovo - } 2 \\
\text { unidades }\end{array}$ & $\begin{array}{l}\text { Café e leite - suco } \\
\text { Queijo - ovo - iogurte }\end{array}$ \\
\hline & $\begin{array}{l}\text { Pão integral mini - } \\
1 \text { unidade }\end{array}$ & $\begin{array}{l}\text { Pão integral mini - } \\
1 \text { unidade }\end{array}$ & $\begin{array}{l}\text { Pão integral mini - } \\
1 \text { unidade }\end{array}$ & $\begin{array}{l}\text { Pão integral mini - } \\
1 \text { unidade }\end{array}$ & $\begin{array}{l}\text { Pão integral mini - } \\
1 \text { unidade }\end{array}$ & $\begin{array}{l}\text { Pão - biscoito água ( } 2 \text { pacotes/ } \\
\text { cuscuz/beiju peq (sem recheio } \\
\text { - colocar a geleia) }\end{array}$ \\
\hline & Geleia diet - 1 und & Geleia diet - 1 und & Geleia diet - 1 und & Geleia diet - 1 und & Geleia diet - 1 und & Raíz, banana terra - fruta \\
\hline & $\begin{array}{l}\text { Raiz cozida ou } \\
\text { banana terra - } 50 \mathrm{~g}\end{array}$ & $\begin{array}{l}\text { Raiz cozida ou } \\
\text { banana terra - } 50 \mathrm{~g}\end{array}$ & $\begin{array}{l}\text { Raiz cozida ou } \\
\text { banana terra - } 100 \mathrm{~g}\end{array}$ & $\begin{array}{l}\text { Raiz cozida ou } \\
\text { banana terra - } 100 \mathrm{~g}\end{array}$ & $\begin{array}{l}\text { Raiz cozida ou } \\
\text { banana terra - } 150 \mathrm{~g}\end{array}$ & \\
\hline & Fruta - $100 \mathrm{~g}$ & Fruta - $100 \mathrm{~g}$ & Fruta $-100 \mathrm{~g}$ & Fruta $-100 \mathrm{~g}$ & Fruta $-100 \mathrm{~g}$ & \\
\hline $\mathrm{CHO}(\mathrm{G})$ & $50 \mathrm{~g}$ & $51 \mathrm{~g}$ & $68 \mathrm{~g}$ & $68 \mathrm{~g}$ & $82 \mathrm{~g}$ & \\
\hline COLAÇÃO & Fruta $-100 \mathrm{~g}$ & Fruta $-100 \mathrm{~g}$ & Fruta $-100 \mathrm{~g}$ & $\begin{array}{l}\text { Vitamina de fruta } \\
\text { c/ leite desnatado - } \\
200 \mathrm{~mL}\end{array}$ & $\begin{array}{l}\text { Vitamina de fruta } \\
\text { c/ leite desnatado - } \\
200 \mathrm{~mL}\end{array}$ & Vitamina iogurte/mingau \\
\hline
\end{tabular}


ilustrações dos modelos dos cardápios com fotos dos pratos e alimentos e um folder ilustrativo com informações sobre a importância da dieta no controle das hiperglicemias hospitalares.

Criação de Ferramentas para Vigilância: Relatórios Periódicos do Cenário Glicêmico e Interconsultoria Virtual

\section{Parecer Virtual da CCGH}

Embora as diretrizes nacionais e internacionais recomendem que os hospitais provenham suporte administrativo para o programa de controle glicêmico, não há padronizações específicas sobre requerimentos infra-estruturais de acordo com o tamanho ou tipo de população assistida no hospital. ${ }^{2} \mathrm{O}$ consenso da American College of Endocrinology e American Diabetes Association preconiza que um time de profissionais de saúde treinado e investimentos diversos em estrutura são componentes para o sucesso de programas como a CCGH. ${ }^{37}$ Entretanto, a falta de recomendações específicas resulta em eventual dificuldade de dimensionamento de tamanho de equipe, especialmente aqueles que farão a assistência dos pacientes diante do número de casos de disglicemias hospitalares.

Para tentar minimizar o problema de insuficiência de equipe versus a prevalência de hiperglicemias no hospital, pesquisadores avaliaram o impacto de uma interconsultoria virtual baseada no prontuário eletrônico.

Mendez e colaboradores ${ }^{38}$ selecionaram, baseados no prontuário eletrônico, pacientes com glicemias $<70 \mathrm{mg} / \mathrm{dL}$ ou $>350 \mathrm{mg} / \mathrm{dL}$ e após revisão dos dados médicos, emitiam uma recomendação médica no PEP. Após a implantação deste modelo (modelo DINGS) houve redução da média glicêmica e percentual de pacientes-dia com glicemias $>350 \mathrm{mg} / \mathrm{dL}$. Rushakoff e colaboradores avaliaram o impacto de uma recomendação de manejo emitida no PEP após avaliação de pacientes com glicemias $<70 \mathrm{mg} / \mathrm{dL}$ e $>225 \mathrm{mg} / \mathrm{dL}$ nas últimas 24 horas e observaram redução de $39 \%$ e $36 \%$ em hiper e hipoglicemias, respectivamente. ${ }^{39}$
Baseada nas evidências, a CCGH do HSI construiu, com a TI, o parecer eletrônico virtual da CCGH. Essa ferramenta importa as glicemias das últimas 36 horas para uma tela de formulário para que o interconsultor virtual, utilizando-se dos dados clínicos do paciente reportados no PEP, emita uma recomendação de conduta baseada nos protocolos institucionais (Figura 9). Um alerta na tela do PEP aparece todas as vezes em que qualquer profissional de saúde abre o prontuário do paciente até que o médico aceite visualizar ou recusar o parecer (Figura 10). O parecer virtual é emitido para todos os pacientes com hiperglicemias críticas, menor que $70 \mathrm{mg} / \mathrm{dL}$ ou acima de $250 \mathrm{mg} / \mathrm{dL}$. Cada parecer emitido também reforça a importância do seguimento dos protocolos, a utilização da prescrição eletrônica de insulinas e a interconsultoria com a equipe de endocrinologia quando o paciente for portador de diabetes.

É importante salientar que o parecer virtual da CCGH não substitui a interconsulta e acompanhamento da endocrinologia hospitalar, e sim complementa o manejo glicêmico por outra forma de cuidado médico. ${ }^{40}$

Substituição dos Glicosímetros por Equipamentos de Acordo com Resolução da ANVISA, RDC 302

Foi detectado que os equipamentos utilizados para mensurar as glicemias capilares utilizados não cumpriam as diretrizes para testes laboratoriais remotos e a normativa da ANVISA, RDC 302. Foi feito estudo de viabilidade e foram substituídos os equipamentos antigos por novos glicosímetros que permitem a rastreabilidade de todo o processo, calibrações, transmissão simultânea para o prontuário eletrônico do paciente e gera dados para análises estatísticas.

\section{Resultados Encontrados e Esperados}

Espera-se que a CCGH contribua para o melhor controle glicêmico, experiência e desfechos de pacientes internados. Espera-se que sejam otimizados indicadores hospitalares como 
Figura 9. Tela do parecer da CCGH no PEP.

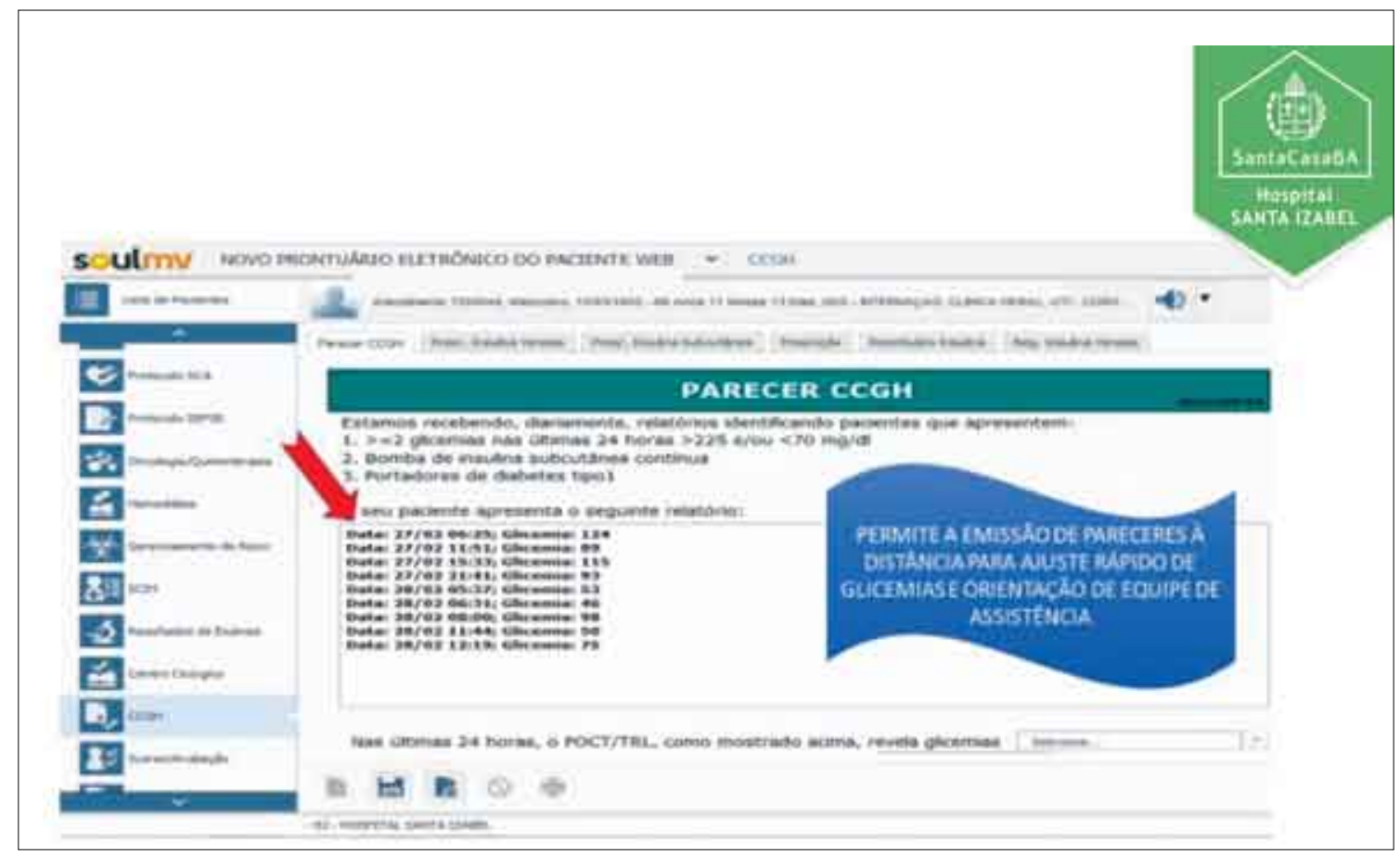

Figura 10. Alerta da CCGH no PEP: parecer da CCGH.

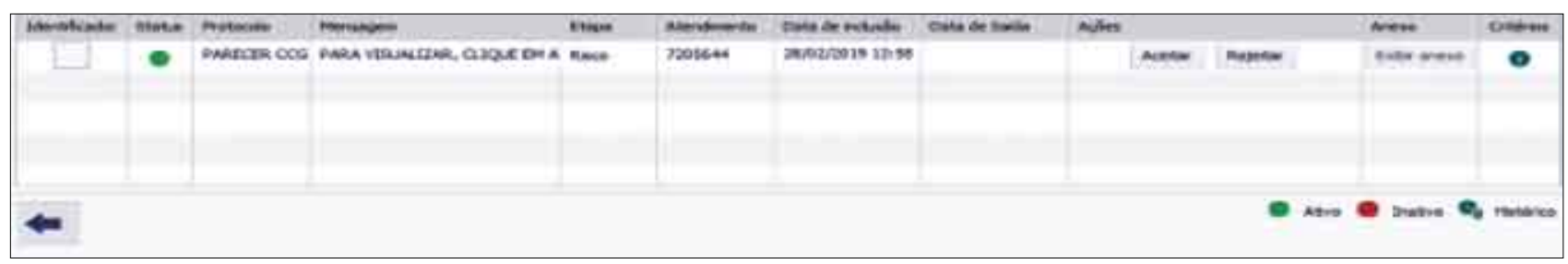

o tempo de internação, redução de infecções e reinternações. Os resultados da implantação já revelam maior conhecimento e segurança das equipes na abordagem das hiperglicemias hospitalares e envolvimento com os protocolos institucionais. A prescrição eletrônica de insulinas e o aviso sonoro da nutrição aumentaram a segurança para o paciente, otimizando os processos de insulinização adequada, enquanto acrescentaram inovação por meio da tecnologia. Dados não publicados mostram melhor média glicêmica e maior proporção de uso do esquema hospitalar basal-bolus de insulina recomendado nas unidades fechadas e redução do tempo de internação hospitalar em unidades abertas nos pacientes hiperglicêmicos que tiveram acompanhamento da CCGH quando internados nas unidades fechadas e acompanhados pela equipe de endocrinologia após a alta da unidade fechada.

\section{Referências}

1. Moghissi ES, Korytkowski MT, DiNardo M, Einhorn D, Hellman R, Hirsch IB, et al. American Association of Clinical Endocrinologists and American Diabetes Association consensus statement on inpatient glycemic control. Diabetes Care. 2009 Jun;32(6):1119-31.

2. Umpierrez GE, Hellman R, Korytkowski MT, Kosiborod M, Maynard GA, Montori VM, et al. Management of hyperglycemia in hospitalized patients in non-critical care setting: an endocrine society clinical practice guideline. J Clin Endocrinol Metab. 2012 Jan;97(1):16-38.

3. Meng Y-Y, Pickett MC, Babey SH, Davis AC, Goldstein H. Diabetes tied to a third of California hospital stays, driving health care costs higher. Policy Brief UCLA Cent Health Policy Res. 2014 May;(PB2014-3):1-7. 
4. Umpierrez GE, Isaacs SD, Bazargan N, You X, Thaler LM, Kitabchi AE. Hyperglycemia: an independent marker of in-hospital mortality in patients with undiagnosed diabetes. J Clin Endocrinol Metab. 2002 Mar;87(3):978-82.

5. Falciglia M, Freyberg RW, Almenoff PL, D'Alessio DA, Render ML. Hyperglycemia-related mortality in critically ill patients varies with admission diagnosis. Crit Care Med. 2009 Dec;37(12):3001-9.

6. Kosiborod M, Rathore SS, Inzucchi SE, Masoudi FA, Wang Y, Havranek EP, et al. Admission glucose and mortality in elderly patients hospitalized with acute myocardial infarction: implications for patients with and without recognized diabetes. Circulation. 2005 Jun 14;111(23):3078-86.

7. Cook CB, Kongable GL, Potter DJ, Abad VJ, Leija DE, Anderson M. Inpatient glucose control: a glycemic survey of 126 U.S. hospitals. J Hosp Med. 2009 Nov;4(9):E7-14.

8. Schmeltz LR, DeSantis AJ, Thiyagarajan V, Schmidt K, O'Shea-Mahler E, Johnson D, et al. Reduction of surgical mortality and morbidity in diabetic patients undergoing cardiac surgery with a combined intravenous and subcutaneous insulin glucose management strategy. Diabetes Care. 2007 Apr;30(4):823-8.

9. Murad MH, Coburn JA, Coto-Yglesias F, Dzyubak S, Hazem A, Lane MA, et al. Glycemic control in noncritically ill hospitalized patients: a systematic review and meta-analysis. J Clin Endocrinol Metab. 2012 Jan;97(1):49-58.

10. Greenberg BH, Abraham WT, Albert NM, Chiswell K, Clare R, Stough WG, et al. Influence of diabetes on characteristics and outcomes in patients hospitalized with heart failure: a report from the Organized Program to Initiate Lifesaving Treatment in Hospitalized Patients with Heart Failure (OPTIMIZE-HF). Am Heart J. 2007 Aug;154(2):277.e1-8.

11. Brodovicz KG, Mehta V, Zhang Q, Zhao C, Davies MJ, Chen J, et al. Association between hypoglycemia and inpatient mortality and length of hospital stay in hospitalized, insulin-treated patients. Curr Med Res Opin. 2013 Feb;29(2):101-7.

12. Cook CB, Castro JC, Schmidt RE, Gauthier SM, Whitaker MD, Roust LR, et al. Diabetes care in hospitalized noncritically ill patients: More evidence for clinical inertia and negative therapeutic momentum. J Hosp Med. 2007 Jul;2(4):203-11.

13. Cook CB, McNaughton DA, Braddy CM, Jameson KA, Roust LR, Smith SA, et al. Management of inpatient hyperglycemia: assessing perceptions and barriers to care among resident physicians. Endocr Pract. 2007 Apr;13(2):117-24.
14. Latta S, Alhosaini MN, Al-Solaiman Y, Zena M, Khasawneh F, Eranki V, et al. Management of inpatient hyperglycemia: assessing knowledge and barriers to better care among residents. Am J Ther. 2011 Sep;18(5):355-65.

15. Moreira ED, Silveira PCB, Neves RCS, Souza C, Nunes ZO, Almeida M da CC, et al. Glycemic control and diabetes management in hospitalized patients in Brazil. Diabetol Metab Syndr. 2013 Oct 18;5(1):62.

16. Maynard G, Berg K, Kulasa K, O’Malley C. The glycemic control implementation guide: Improving glycemic control, preventing hypoglycemia and optimizing care of the inpatient with hyperglycemia and diabetes [Internet]. Society of Hospital Medicine. 2015 [cited 2019 May 30]. Available from: http://www. hospitalmedicine.org/gc.

17. American Diabetes Association. 14. Diabetes Care in the Hospital: Standards of Medical Care in Diabetes2018. Diabetes Care. 2018;41(Suppl 1):S144-51.

18. Yatabe T, Inoue S, Sakaguchi M, Egi M. The optimal target for acute glycemic control in critically ill patients: a network meta-analysis. Intensive Care Med. 2017 Jan;43(1):16-28.

19. Umpierrez GE, Smiley D, Jacobs S, Peng L, Temponi A, Mulligan P, et al. Randomized study of basalbolus insulin therapy in the inpatient management of patients with type 2 diabetes undergoing general surgery (RABBIT 2 surgery). Diabetes Care. 2011 Feb;34(2):256-61.

20. Bansal V, Mottalib A, Pawar TK, Abbasakoor N, Chuang E, Chaudhry A, et al. Inpatient diabetes management by specialized diabetes team versus primary service team in non-critical care units: impact on 30-day readmission rate and hospital cost. BMJ Open Diabetes Res Care. 2018 Apr 5;6(1):e000460.

21. Helmle KE, Dechant AL, Edwards AL. Implementation of a Multidisciplinary Educational Strategy Promoting Basal-Bolus Insulin Therapy Improves Glycemic Control and Reduces Length of Stay for Inpatients With Diabetes. Clin Diabetes. 2019 Jan;37(1):82-5.

22. AACE AACE. Clinical Evidence for Glucose Control in the Inpatient Setting. [Internet]. Clinical Evidence for Glucose Control in the Inpatient Setting. [cited 2019 May 28]. Available from: http://inpatient.aace. com/rationale-for-achieving-glycemic-control.

23. Draznin B, Gilden J, Golden SH, Inzucchi SE, PRIDE investigators, Baldwin D, et al. Pathways to quality inpatient management of hyperglycemia and diabetes: a call to action. Diabetes Care. 2013 Jul;36(7):1807-14.

24. Christensen MB, Gotfredsen A, Nørgaard K. Efficacy of basal-bolus insulin regimens in the inpatient management of non-critically ill patients with type 2 diabetes: A systematic review and meta-analysis. Diabetes Metab Res Rev. 2017 Feb 23;33(5). 
25. Pacienty Safety Network PSN. Medication errors with the dosing of insulin: problems across the continuum. PA-PSRS Patient Saf Advis. 2010 Mar.

26. SBD SB de D. Controle da Glicemia do Paciente Hospitalizado. O. Posicionamento Oficial SBD [Internet]. www.diabetes.org.br. 2015 [cited 2019 May 30]. Available from: https://www.diabetes.org.br/ publico/images/2015/pdf.

27. Puig J, Supervía A, Márquez MA, Flores J, Cano JF, Gutiérrez J. Diabetes team consultation: impact on length of stay of diabetic patients admitted to a short-stay unit. Diabetes Res Clin Pract. 2007 Nov;78(2):211-6.

28. Flanagan D, Moore E, Baker S, Wright D, Lynch P. Diabetes care in hospital--the impact of a dedicated inpatient care team. Diabet Med. 2008 Feb;25(2):147-51.

29. Levetan CS, Salas JR, Wilets IF, Zumoff B. Impact of endocrine and diabetes team consultation on hospital length of stay for patients with diabetes. Am J Med. $1995 \mathrm{Jul} ; 99(1): 22-8$.

30. Mathioudakis N, Pronovost PJ, Cosgrove SE, Hager D, Golden SH. Modeling inpatient glucose management programs on hospital infection control programs: an infrastructural model of excellence. Jt Comm J Qual Patient Saf. 2015 Jul;41(7):325-36.

31. Braithwaite SS, Magee M, Sharretts JM, Schnipper JL, Amin A, Maynard G, et al. The case for supporting inpatient glycemic control programs now: the evidence and beyond. J Hosp Med. 2008 Sep;3(5 Suppl):6-16.

32. Câmara-de-Souza A. InsulinAPP-UTI [Internet]. InsulinAPP-UTI. 2017 [cited 2019 May 27]. Available from: http://www.tack.com.br/insulinappbeta/

33. Souza ABC, Cukier P, Toyoshima MT, Oliveira E, Di Rienzo RT, Nery M. InsulinAPP-ICU: a safe and effective digital plataform for continuous intravenous insulin therapy in intensive care unit. Rev Bras Ter Intensiva. 2017 Nov;29(Suppl. 1):S209-210.
34. Aspden L Cronenwett LR, Wolcott J, Bootman JL, Cronenwett LR. Preventing Medication Errors: Quality Chasm Series. Committee on Identifying and Preventing Medication Errors, editor. Washington, DC: The National Academies Press; 2006 Jul.

35. Gillaizeau F, Chan E, Trinquart L, Colombet I, Walton RT, Rège-Walther $\mathrm{M}$, et al. Computerized advice on drug dosage to improve prescribing practice. Cochrane Database Syst Rev. 2013 Nov 12;(11):CD002894.

36. Maynard G, Kulasa K. Addressing Inpatient Diabetes: A QI Perspective Special Focus on Hypoglycemia Reduction [Internet]. Addressing Inpatient Diabetes: A QI Perspective Special Focus on Hypoglycemia Reduction. [cited 2019 May 30]. Available from: https://slideplayer.com/ slide/6428970/\#.XPVvZ9I-QAs.gmail

37. ACE/ADA Task Force on Inpatient Diabetes. American College of Endocrinology and American Diabetes Association consensus statement on inpatient diabetes and glycemic control. Endocr Pract. 2006 Aug;12(4):458-68.

38. Mendez CE, Ata A, Rourke JM, Stain SC, Umpierrez G. Daily inpatient glycemic survey (dings): a process to remotely identify and assist in the management of hospitalized patients with diabetes and hyperglycemia. Endocr Pract. 2015 Aug;21(8):927-35.

39. Rushakoff RJ, Sullivan MM, MacMaster HW, Shah AD, Rajkomar A, Glidden DV, et al. Association between a virtual glucose management service and glycemic control in hospitalized adult patients: an observational study. Ann Intern Med. 2017 May 2;166(9):621-7.

40. Drincic AT, Akkireddy P, Knezevich JT. Common models used for inpatient diabetes management. Curr Diab Rep. 2018 Feb14;18(3):10. 